\title{
Dystrophic Calcifications in the Breast from Secondary Hyperparathyroidism
}

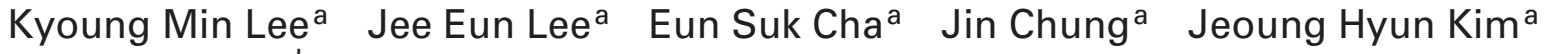 \\ Byung In Moon ${ }^{b}$ \\ a Department of Radiology, Ewha Womans University, School of Medicine, Seoul, Republic of Korea; \\ ${ }^{b}$ Department of Surgery, Ewha Womans University, School of Medicine, Seoul, Republic of Korea
}

\section{Established Facts}

- Soft tissue calcification is common in patients with secondary hyperparathyroidism, but calcifications in the breast parenchyma are not common.

\section{Novel Insights}

- We report an uncommon case of a woman with large breast calcifications from secondary hyperparathyroidism.

\section{Keywords}

Breast · Calcinosis · Calcification · Hyperparathyroidism

\section{Summary}

Background: Soft tissue calcification is common in patients with secondary hyperparathyroidism who have received long-term treatment with hemodialysis. However, calcifications in the breast parenchyma are not common. We report a case of a woman with dystrophic breast calcifications from secondary hyperparathyroidism. Case Report: A 65-year-old woman presented with a palpable mass in her right breast which she had discovered 1 month ago. She had a medical history of end-stage renal disease. Mammography and ultrasound revealed large dystrophic calcifications in both breasts. Core needle biopsy was performed for calcifications in the right breast, and the pathologic diagnosis was dystrophic calcification in the stroma from secondary hyperparathyroidism. Conclusion: Reviewing our case will contribute to a fast and correct diagnosis in patients with dystrophic breast calcifications and lab results indicating secondary hyperparathyroidism, and will help discriminate these benign lesions from malignancies.

(c) 2018 S. Karger GmbH, Freiburg

\section{Introduction}

Soft tissue calcification is a common finding in patients with secondary hyperparathyroidism who have received long-term treatment with hemodialysis. These calcifications tend to deposit in the tunica media of arteries, the cornea and conjunctiva of the eyes, paraarticular soft tissues, skin, and subcutaneous tissues; however, metastatic calcifications in the breast parenchyma are not common [1].

We report a case of a woman with dystrophic breast calcifications from secondary hyperparathyroidism who presented with a palpable breast mass and had undergone maintenance hemodialysis for 8 years.

\section{KARGER}

Fax +497614520714

\section{(๑) 2018 S. Karger GmbH, Freiburg}




\section{Case Report}

A 65-year-old woman presented with a palpable mass in her right breast which she had discovered 1 month ago. She had a medical history of hypertension, diabetes mellitus, and coronary artery disease. She was also diagnosed with end-stage renal disease (ESRD) 18 years ago and had undergone maintenance hemodialysis 3 times a week for 8 years.

Physical examination revealed an approximately 3-cm fixed mass in her right upper central breast. Laboratory evaluation revealed hyperphosphatemia (serum ionized phosphorus, $8.8 \mathrm{mg} / \mathrm{dl}$; normal range, $2.3-4.5 \mathrm{mg} / \mathrm{dl}$ ), hyperparathyroidism (serum parathyroid hormone (PTH), $606 \mathrm{pg} / \mathrm{ml}$; normal range, $15-65 \mathrm{pg} / \mathrm{ml}$ ), and a normal serum calcium level. Routine mammography was performed. A large dystrophic calcification was noted in the upper outer quadrant of her right breast. There were multiple grouped dystrophic, large rod-like, coarse calcifications in her left breast (fig. 1). Bilateral whole-breast ultrasound was performed on the same day. Breast ultrasound revealed multiple parenchymal calcifications with posterior shadowing in the upper portion of both breasts (fig. 2). Chest radiographies were retrospectively reviewed. Small dense calcifications were first detected in her right breast on a chest radiograph performed 27 months ago, and they had markedly increased in size within 12 months. Although the routine mammography and ultrasound showed typical dystrophic calcifications, clinicians wanted to perform a biopsy to rule out the possibility of

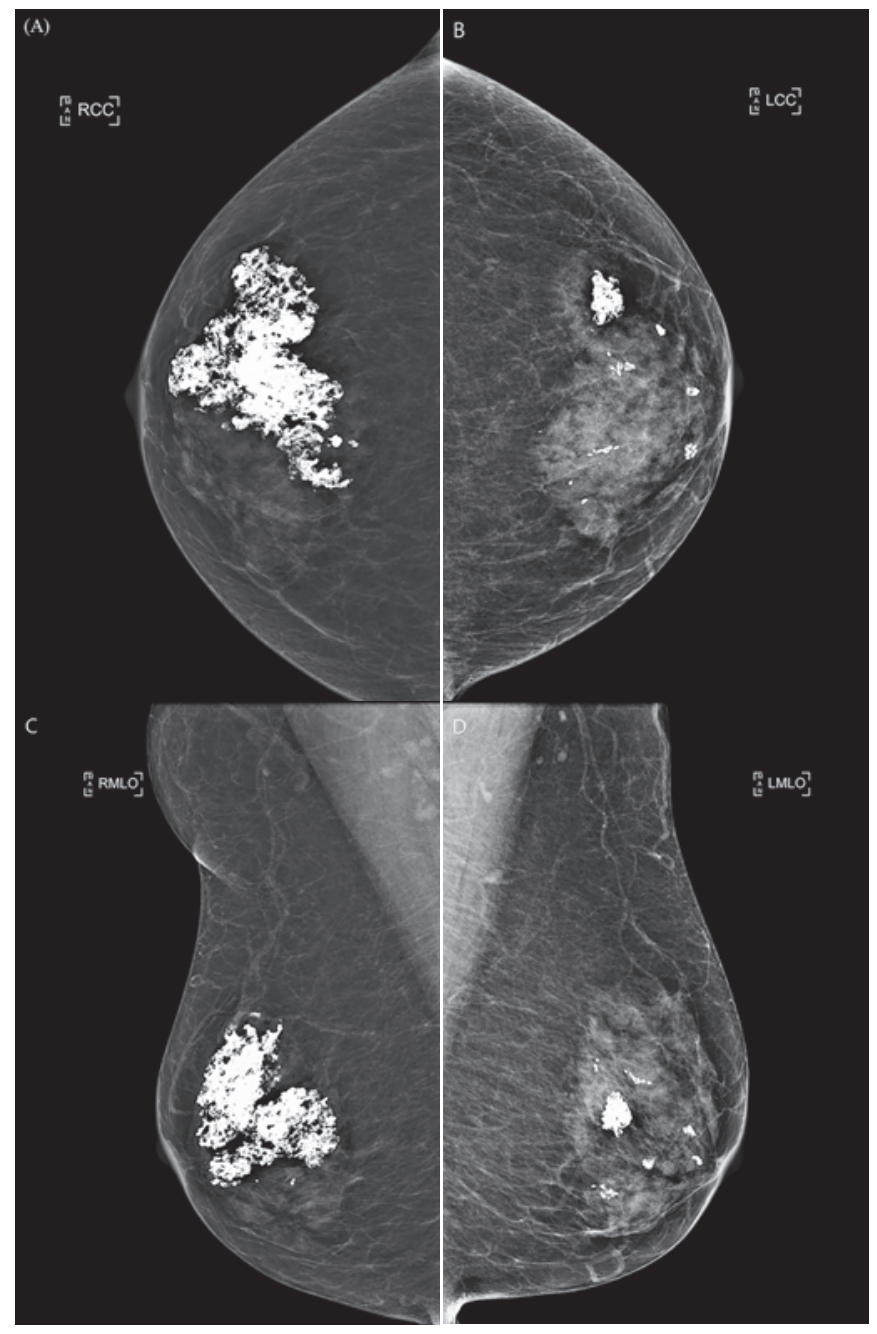

Fig. 1. Digital mammography with both cranial-caudal (CC) and mediolateral-oblique (MLO) views demonstrate a large dystrophic calcification in the upper outer quadrant of the right breast. Multiple grouped dystrophic, large, rod-like calcifications are also noted in the left breast. breast malignancy since the patient presented with a palpable mass. Core needle biopsy was performed for calcifications in the upper portion of the right breast using a 14-gauge needle.

The pathologic diagnosis was dystrophic calcification in the stroma. Considering the patient's medical history, the final diagnosis was dystrophic calcification in the breast from secondary hyperparathyroidism induced by chronic kidney disease (CKD). After 6 months, a follow-up mammography was performed. Mammography showed increased density and size of the previous dystrophic calcifications in both breasts. Laboratory tests still showed hyperphosphatemia (serum ionized phosphorus, $7.5 \mathrm{mg} / \mathrm{dl}$ ), hyperparathyroidism (serum PTH, $666.80 \mathrm{pg} / \mathrm{ml}$ ), and a normal serum calcium level. For the treatment of secondary hyperparathyroidism, paricalcitol (Zemplar ${ }^{\circledR}$; AbbVie Inc., Bluff Lake, IL, USA) and phosphorus binders were prescribed.

\section{Discussion}

It is known that patients with CKD or ESRD develop uremic calcification in various organs and tissues owing to disturbances in the calcium, phosphorus, and PTH metabolism [2]. Several studies have suggested that women with CKD or ESRD have a higher prevalence of breast calcifications. The majority of calcifications were typically benign, such as vascular calcifications, ductal calcifications located behind the areola or typically oriented toward the nipple, and small, oval, or scattered parenchymal calcifications [3-5].

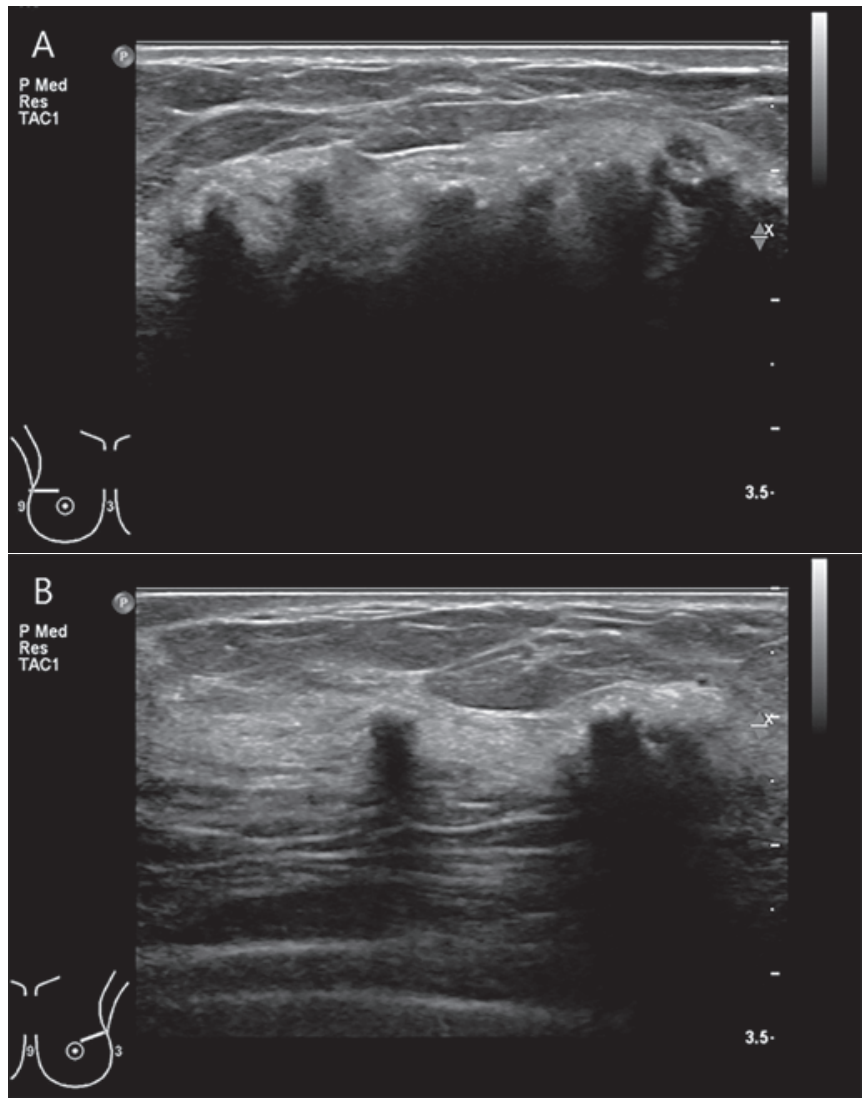

Fig. 2. Ultrasonography of $\mathbf{A}$ right and $\mathbf{B}$ left breast showing large parenchymal calcification with posterior shadowing in the upper portion of the right breast (palpable lesion). Multiple small parenchymal calcifications are seen in the upper portion of the left breast. 
Soft tissue calcification is a common finding in patients with secondary hyperparathyroidism resulting from CKD. CKD leads to hyperphosphatemia and decreased serum calcium due to impaired renal clearance. This stimulates PTH secretion, leading to secondary hyperparathyroidism. With respect to the pathogenesis of metastatic soft tissue calcification, it is known that an increase in the calcium phosphate product leads to calcium deposition in the tunica media of the arteries, the periarticular and subcutaneous tissue, and multiple visceral organs [6]. Ectopic soft tissue calcification in the breast has only been described in 4 case reports. Diffuse calcification in the ductal and glandular tissue of the breast in a woman on maintenance hemodialysis for ESRD was first reported in 1977, and it was detected on routine chest radiography after the start of dialysis [7]. Follow-up radiography showed considerable resolution of the breast calcifications. In 1996, Resnikoff et al. [8] reported numerous coarse calcifications predominantly in the right breast in a woman with CRF, which were detected on computed tomography of the chest performed for confirmation of a nodule that looked suspicious on chest radiography. Unlike these 2 case reports, the other 2 cases presented with a palpable breast mass. 1 report described a case presenting with a solitary, nontender mass in left breast. The mammographic image showed diffuse calcifications in both breasts, predominantly located in a single breast quadrant which was the palpable site [9]. In the most recent case, which was reported in 2003 by Cowlam and Bucknall [10], the patient presented with bilaterally tender, lumpy breasts. She was diagnosed with ectopic calcifications of the breasts. Subcutaneous nodules were also noted around the ileostomy (after surgery for Crohn's disease) and abdominal wall. We reviewed the mammographic findings of previous cases and concluded that the common finding associated with metastatic breast calcifications was coarse and dense calcifications in the glandular parenchyma (symptomatic or asymptomatic).

The treatment options for calcifications suggested in previous reports include dietary phosphate restriction, phosphate binder treatment, intensified dialysis treatment, dialysis using a low-calcium dialysate, parathyroidectomy in those with high PTH levels, and surgical excision of the calcified mass [11]. Among the 4 case reports, only 1 showed considerable resolution of the breast calcifications 20 months after initiation of hemodialysis [7]. In our case, mammographic follow-up was not performed, but increased calcification in both breasts was detected on chest radiography after 2 years.

In conclusion, dystrophic calcifications in the breast from secondary hyperparathyroidism are not common. To the best of our knowledge, there are only 4 reported cases. In previous reports, images were obtained by conventional mammography, while in our case a good-quality image was obtained by digital mammography. The patient described in our case shows mammographic and pathologic findings of uncommon dystrophic breast calcifications from secondary hyperparathyroidism. Information obtained from our case may help clinicians and radiologists to make a fast and correct diagnosis in patients with dystrophic breast calcifications and lab results indicating secondary hyperparathyroidism, and will help discriminate these benign lesions from malignancies.

\section{Disclosure Statement}

The authors of this manuscript declare no relationship with any companies whose products or services may be related to the subject matter of the article. The authors declared that no competing interest exists.

\section{References}

1 Massry SG, Coburn JW, Popovtzer MM, Shinaberger JH, Maxwell MH, Kleeman CR: Secondary hyperparathyroidism in chronic renal failure: the clinical spectrum in uremia, during hemodialysis, and after renal transplantation. Arch Intern Med 1969;124:431-441.

2 Block GA, Port FK: Re-evaluation of risks associated with hyperphosphatemia and hyperparathyroidism in dialysis patients: recommendations for a change in management. Am J Kidney Dis 2000;35:1226-1237.

3 Evans A, Cohen M, Cohen G: Patterns of breast calcification in patients on renal dialysis. Clin Radiol 1992, 45:343-344.

4 Castellanos M, Varma S, Ahern K, Grosso S-J, Buchbinder S, D’Angelo D, Raia C, Kleiner M, Elsayegh S: Increased breast calcifications in women with ESRD on dialysis: implications for breast cancer screening. Am J Kidney Dis 2006;48:301-306.
5 Sivakumar V, Rani Ch S, Lakshmi AY, Lakshmi BV: Breast calcifications in women with end-stage renal disease on maintenance hemodialysis. Saudi J Kidney Dis Transpl 2011;22:142-145.

6 Cooper RA, Berman S: Extensive breast calcification in renal failure. J Thorac Imaging 1988;3:81-82.

7 Han SY, Witten DM: Diffuse calcification of the breast in chronic renal failure. AJR Am J Roentgenol 1977; 129:341-342.

8 Resnikoff LB, Mendelson EB, Tobin CE, Hendrix TM: Breast imaging case of the day. Metastatic calcification in the breast from secondary hyperparathyroidism induced by chronic renal failure. Radiographics 1996;16: 1512-1513.
9 Evans SE, Whitehouse GH: Extensive calcification in the breast in chronic renal failure. Br J Radiol 1991;64: 757-759.

10 Cowlam TE, Bucknall TE: Cutaneous ectopic breast calcification in a haemodialysis patient. Breast 2003;12: 342-344.

11 Lacativa PGS, Castagnaro M, Patricio PJM, de Farias MLF: Ectopic calcifications in end-stage renal failure due to different mechanisms. Clin Radiol Extra 2004; 59:120-124. 